\title{
Fermilab main injector: High intensity operation and beam loss control
}

\author{
Bruce C. Brown, ${ }^{*}$ Philip Adamson, David Capista, Weiren Chou, Ioanis Kourbanis, \\ Denton K Morris, Kiyomi Seiya, Guan Hong Wu, and Ming-Jen Yang \\ Accelerator Division, Fermi National Accelerator Laboratory, Batavia, Illinois 60510, USA
}

(Received 1 December 2012; published 9 July 2013)

\begin{abstract}
From 2005 through 2012, the Fermilab Main Injector provided intense beams of $120 \mathrm{GeV}$ protons to produce neutrino beams and antiprotons. Hardware improvements in conjunction with improved diagnostics allowed the system to reach sustained operation at $400 \mathrm{~kW}$ beam power. Transmission was very high except for beam lost at or near the $8 \mathrm{GeV}$ injection energy where $95 \%$ beam transmission results in about $1.5 \mathrm{~kW}$ of beam loss. By minimizing and localizing loss, residual radiation levels fell while beam power was doubled. Lost beam was directed to either the collimation system or to the beam abort. Critical apertures were increased while improved instrumentation allowed optimal use of available apertures. We will summarize the improvements required to achieve high intensity, the impact of various loss control tools and the status and trends in residual radiation in the Main Injector.
\end{abstract}

DOI: 10.1103/PhysRevSTAB.16.071001

PACS numbers: 29.20.dk, 29.27.Fh, 41.85.Ja, 41.85.Si

\section{PROTONS TO PRODUCE NEUTRINOS AND ANTIPROTONS}

On April 30, 2012, the Fermilab accelerator complex began an extended shutdown for facility upgrades. This followed seven months after the end of operation for the Tevatron on September 30, 2011, with the accompanying end of Antiproton Source operation. For the Fermilab Main Injector, this marked 13.5 years of commissioning and operation in successively higher intensity operation modes. As the physics program requirements demanded more beam power, limitations in the intensity and beam quality from the Fermilab Booster were overcome by using slip stacking injection [1]. This was implemented first for antiproton (pbar) production and later for neutrino production as well. Once this concept was proven, required upgrades to the Linac, Booster, and Main Injector to support high intensity operation were identified and a Proton Plan [2,3] organized to implement them. As intensities increased, a program of monitoring and mitigating losses and residual radiation has controlled the radiation exposure for personnel involved in maintenance and upgrade activities.

Figure 1 illustrates this intensity increase using the number of protons per cycle on a periodic sample of the acceleration cycles. An injection from the Booster is termed a "batch" with typical intensity of $4-5 \times 10^{12}$ protons and up to $84 \mathrm{rf}$ buckets of beam. Machine commissioning was followed by multibatch operation for a

\footnotetext{
*bcbrown@fnal.gov.

Published by the American Physical Society under the terms of the Creative Commons Attribution 3.0 License. Further distribution of this work must maintain attribution to the author(s) and the published article's title, journal citation, and DOI.
}

Tevatron fixed-target run. In 2001, this transitioned to a Tevatron collider run which utilized a single batch from the Booster for pbar production. Slip stacking injection of two Booster batches for pbar production became operational in December 2004. In May 2005, the NuMI (Neutrinos at the Main Injector) beam line for neutrino production began operation which required each acceleration cycle to provide 5 batches to be sent to the NuMI target. Mixed mode slip stacking injection became operational in August 2005 with 5 batches to be sent to NuMI plus a double (slip stacked) batch for pbar production $(5+2$ cycle). Eleven batch mixed mode slip stacking $(9+2$ cycle) that provides four double batches for increased NuMI beam was commissioned in January 2008 at the same time as was the Main Injector collimation system [4]. At that point, intensity was limited by losses in both the Main Injector and the Booster. Collimation, along with improved Booster beam quality, controlled activation and permitted Main Injector intensity per cycle to increase.

Several other features of the Fermilab High Energy Physics (HEP) program are apparent in Fig. 1. Facility upgrades are accomplished using shutdown periods of several weeks. Periods of reduced intensity mark Tevatron failures or the time required to repair or replace the NuMI horn or target. When pbar production ended, intensity ceilings were needed to limit neutrino target thermal shock. Accelerating cycles with nine batches, including three which were slip stacked, provided the required intensity. The reduced per pulse intensity from October 2011 through April 2012 reflects this limitation. This figure reports measurements from older instrumentation or data recording for which improved systems were available by 2007 and spikes above the trend are typically due to instrumentation or data recording errors. 


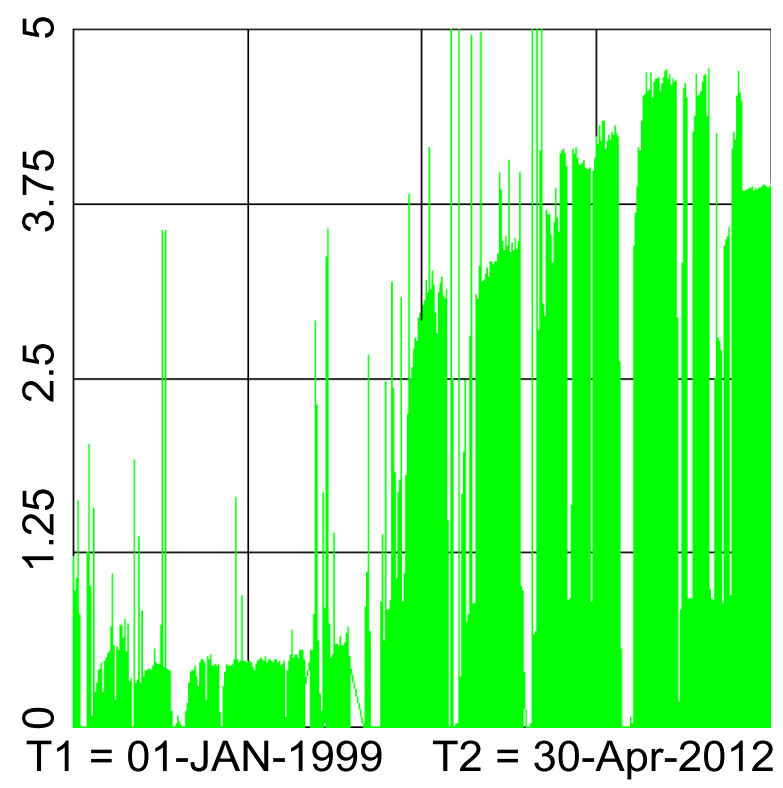

FIG. 1. Sampled intensity per cycle from January 1999 through April 2012 with full scale of $5 \times 10^{13}$ protons per pulse (50 Tp per pulse). Vertical grid lines are at May 2, 2002, August 31, 2005, and December 30, 2008.

\section{INTRODUCTION TO THE MAIN INJECTOR}

The Fermilab Main Injector Project [5] was created to enhance the physics capabilities of the Tevatron collider and to provide beams of $120 \mathrm{GeV}$ protons for test beams and fixed-target particle physics experiments. The initial goal for high intensity operation was $3 \times 10^{13}$ protons per pulse at $120 \mathrm{GeV}$. Construction of the Main Injector began in June 1992 with commissioning beginning in September 1998. Basic properties of the Main Injector and important features for high intensity operation are summarized in Table I. With approval of the NuMI neutrino beam and the MINOS experiment, high intensity operation became the focus for upgrades.

In addition to providing $120 \mathrm{GeV}$ protons at high intensity, the Main Injector was required to accelerate high quality beams of protons and antiprotons to $150 \mathrm{GeV}$ for injection to the Tevatron, to supply $120 \mathrm{GeV}$ protons to fixed-target experiments and test beams, and to transfer antiproton beams at $8 \mathrm{GeV}$ to and from the permanent magnet Recycler Ring in the same tunnel and from the Antiproton Source. Transfers of $8 \mathrm{GeV}$ protons were required for tuning the transfer lines and the Accumulator and Debuncher (Antiproton Source).

This document is devoted to the high intensity operation [6]. We will describe instrumentation improvements and the residual radiation monitoring program. An overview of slip stacking injection and $\mathrm{rf}$ modifications required to achieve high per pulse intensities will be provided. Dampers needed to control instabilities, aperture improvements which reduce losses, and collimation systems to
TABLE I. Main Injector properties including typical beam properties for high intensity $120 \mathrm{GeV}$ operation.

\begin{tabular}{|c|c|c|}
\hline \multicolumn{3}{|l|}{ Lattice properties } \\
\hline Measured circumference & 3319.4151 & $\mathrm{~m}$ \\
\hline Courant-Snyder amplitude $\beta_{\max }$ & 57 & $\mathrm{~m}$ \\
\hline Courant-Snyder amplitude $\beta_{\min }$ & 10 & $\mathrm{~m}$ \\
\hline Maximum dispersion function & 1.9 & $\mathrm{~m}$ \\
\hline Transverse admittance & $>40 \pi$ & $\mathrm{mm}-\mathrm{mr}$ \\
\hline Longitudinal admittance & $>0.5$ & $\mathrm{eVs}$ \\
\hline Nominal horizontal tune & 26.425 & \\
\hline Nominal vertical tune & 25.415 & \\
\hline Natural horizontal chromaticity & -33.6 & \\
\hline Natural vertical chromaticity & -33.9 & \\
\hline Transition $\gamma$ & 21.8 & \\
\hline \multicolumn{3}{|l|}{ rf system properties } \\
\hline Booster harmonic number & 84 & \\
\hline Main injector harmonic number & 588 & \\
\hline Injection frequency & 52.811 & $\mathrm{MHz}$ \\
\hline Extraction frequency & 53.104 & $\mathrm{MHz}$ \\
\hline Accelerating cavities & 18 & \\
\hline Peak voltage & 4 & MV \\
\hline Maximum acceleration rate & 204 & $\mathrm{GeV} / \mathrm{s}$ \\
\hline \multicolumn{3}{|l|}{ Nominal injected beam properties } \\
\hline Kinetic energy & 8 & $\mathrm{GeV}$ \\
\hline Transverse emittance (95\%) & $15 \pi$ & $\mathrm{mm}-\mathrm{mr}$ \\
\hline $\begin{array}{l}\text { Longitudinal emittance } \\
\text { per bunch }(95 \%)\end{array}$ & 0.08 & $\mathrm{eVs}$ \\
\hline Momentum spread ( $\delta p 95 \%)$ & 8 & $\mathrm{MeV} / \mathrm{c}$ \\
\hline Bunches transferred per & 81 & \\
\hline
\end{tabular}

Mixed mode operation-pbar beam

Booster beam intensity

Number of batches

Transmission efficiency

Extracted beam intensity

Mixed mode operation-NuMI beam

Booster beam intensity

Number of batches

Transmission efficiency

Extracted beam intensity

Slip stack frequency difference

Slip stack interval

Typical extracted intensity $(\mathrm{NuMI}+$ pbar)

Record extracted intensity

Main Injector cycle time (mixed mode)

Beam power (typical)

Beam power (record for $1 \mathrm{~h}$ ) $\begin{array}{cc}4.3 \times 10^{12} & \text { protons/batch } \\ 2 & \\ 93 \% & \\ 8 \times 10^{12} & \text { protons } / \text { cycle }\end{array}$

$4 \times 10^{12}$ protons/batch 9

$95 \%$

$34 \times 10^{12}$ protons/cycle

$1430 \quad \mathrm{~Hz}$

$5 / 15$ seconds

$42 \times 10^{12}$ protons/cycle

$46.3 \times 10^{12}$ protons/cycle

2.2 seconds

$380 \quad$ kWatts

$400 \quad$ kWatts

localize the remaining beam loss will be discussed. We will describe minor problems which were resolved so that we achieved low residual radiation nearly everywhere in the Main Injector enclosure. The beam power and proton intensities delivered will be summarized. 


\section{UPGRADES FOR HIGH INTENSITY: INSTRUMENTATION, MONITORING, AND CONTROL}

The instrumentation for commissioning the Main Injector used data acquisition and electronic systems developed for the Fermilab Main Ring in the 1980s. By 2006 new systems were commissioned. The new beam position monitor (BPM) system employs digital signal receivers for signal conversion. Enhanced flexibility as well as improved resolution for position measurement are available [7]. For the 250 ionization chamber beam loss monitors (BLM's) [8], a new digitization and data recording system [9] provides flexibility for studies and much enhanced monitoring capability. A more sophisticated data collection system from the existing beam current monitors [dc current transformers (DCCT)] was developed using a standalone microprocessor (BEAMS front end [10]). Together these new instruments allowed a more systematic study of the machine and improved displays of routine operation.

New control console programs were developed to employ the BLM system [11]. For studies, a flexible system to set data collection times provided details about loss mechanisms by allowing time correlated measurements on all loss monitors. Beam loss displays were particularly significant for improving the overall loss pattern by emphasizing high losses while disclosing lesser beam loss locations that had previously gone unobserved. Figure 2 shows beam losses for operations in January 2009. This display occupies a prominent place in the Fermilab Accelerator Control Room.

Injection period loss (blue) are seen all around the ring. Those in the injection region (green brace) are created by circulating beam in the injection gap. Beam lost during early acceleration (yellow) are due to unaccelerated (uncaptured) beam (as described in Sec. IV B). By this time (2009), the collimation system (see Sec. IV F) was beginning to localize these losses at the collimator region (cyan brace) but they are still seen in many other locations. Further efforts were required. End of cycle losses (green), when not overlaid by earlier loss integrals, are typically from the extraction process. They are apparent at both Recycler transfer regions (red brace), at the abort location (yellow brace), and the high energy transfer locations (purple brace). Other locations with no special lattice function also show loss. Figure 3, from 2011, illustrates the progress documented in this paper. These results are discussed further in Sec. VIII A. Many losses were reduced by only employing proper orbit correction.

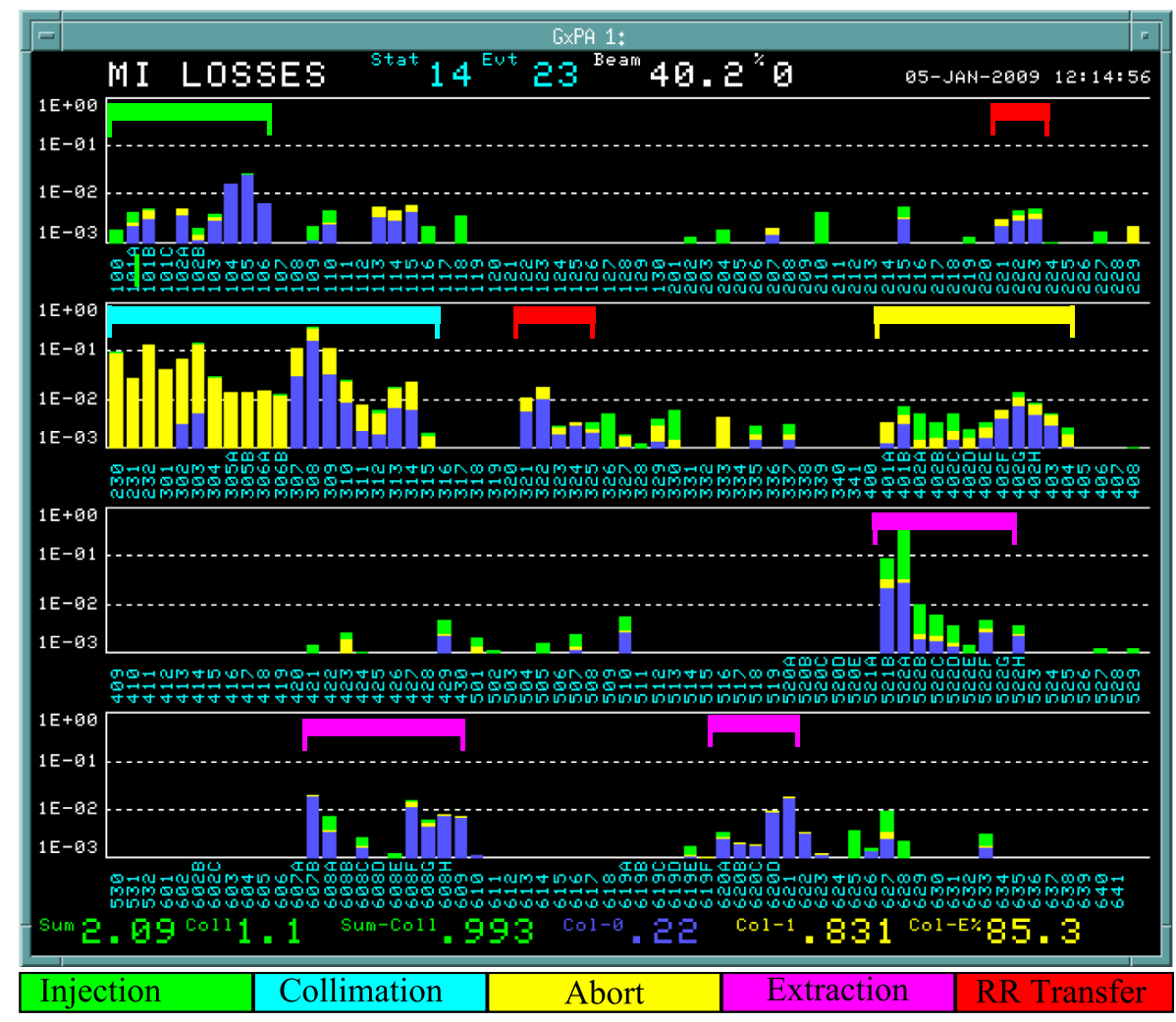

FIG. 2. Beam loss monitor display from a Main Injector cycle in January 2009 at an intensity of $4.4 \times 10^{13}$ protons. The three decade logarithmic vertical scale in rads/pulse ranges from 1 millirad to $1 \mathrm{rad}$. Values shown are integrated loss at each BLM in the ring at the end of the cycle in green, overlaid in yellow by loss integral after $1.5 \%$ acceleration, then overlaid in blue by the loss integral at the end of injection. In typical operation, most of the green loss results from the extraction process at the very end of the cycle. Functional areas of the ring are marked by colored braces that are identified below the display. 


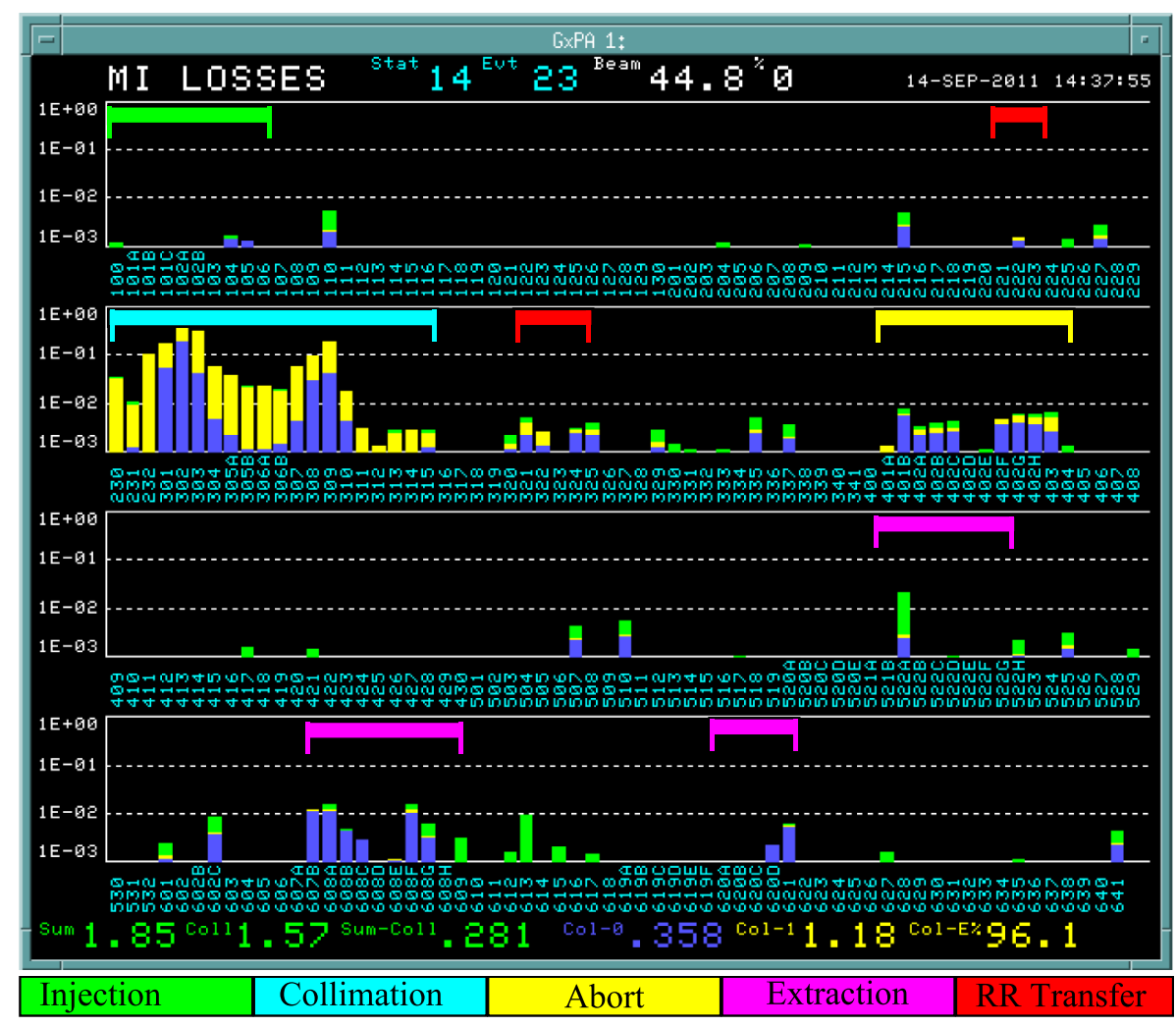

FIG. 3. Beam loss monitor display from Main Injector cycle in September 2011 at an intensity of $4 \times 10^{13}$ protons prior to the end of pbar production. Loss monitors are shown sequentially around the Main Injector. Compare to Fig. 2 above. Some remaining BLM signals are due to pedestal offset, not beam loss.

Preparations for the high intensity operation for neutrino production included a program to identify residual radiation issues in the Main Injector tunnel. Exploratory residual radiation measurements in 2004 and 2005 monitored more than 100 locations with more than 20 millirad/hr residual radiation on contact. By October 2005, a program using a sensitive meter to monitor 127 (later expanded to 142) barcoded locations was initiated [12]. Loss issues at beam transfer regions were monitored and some unexpected loss patterns were identified and explored (see Sec. V B). The need for loss localization using collimators was documented. See Sec. VIII B and Fig. 13 for some results from these measurements.

\section{A. Injection line collimation}

The exploratory residual radiation monitoring program included detailed studies of the radiation pattern that suggested that beam halo was greatly increasing the number of radioactive locations. Collimation of the Booster beam in the transfer line was an obvious option. In order to collimate beam in a transfer line, in both horizontal and vertical planes it is necessary to have collimation edges on two sides of the beam and at two locations. This was accomplished in the Fermilab Booster to Main Injector transfer line (MI8 Line) with the corners of four rectangular apertures using pairs of collimators at two locations separated by $90^{\circ}$ phase advance. This collimation system [13] was installed in 2006 and has operated to scrape beam edges beyond about $99 \%$ of the beam. Beam orbit drift would cause fluctuations in the transmitted beam by asymmetric collimation. This was controlled by an autotune system with frequent beam position measurements to determine new trim magnet settings resulting in stability at the $\sim 0.1-0.2 \mathrm{~mm}$ level.

\section{B. Instability control for high intensity operation}

Commissioning of the Main Injector achieved a goal of accelerating $2 \times 10^{13}$ protons per cycle. However, this required use of large negative chromaticity to control the resistive wall instability. The resulting beam lifetime at injection energy resulted in beam losses of $10 \%$. This loss was alleviated by using the transverse mode damping provided by a digital damper system [14]. Very high transmission is achieved with near-zero chromaticity when using the digital dampers.

Longitudinal damping by this system improved the longitudinal emittance by damping injection oscillations from the Booster and by avoiding coupled bunch instabilities in the Main Injector seeded by the Booster oscillations. This smaller emittance is important in achieving the 
shortest possible bunch length for efficient pbar production. Longitudinal control was also important for Tevatron injection. The longitudinal dampers improved the efficiency of slip stacking (see Sec. IV below) by $1 \%$ permitting higher intensities and also modestly improved the ability to control losses at Main Injector transition.

\section{SLIP STACKING TO ACHIEVE HIGH INTENSITY}

The 40-year-old Fermilab Booster provides the $8 \mathrm{GeV}$ beam injected into the Main Injector. The Main Injector circumference is 7 times that of the Booster ${ }^{1}$ but the need for clean transfers limits operation to six Booster batches, leaving time for the rise and fall of the fields in the transfer kicker magnets (kicker gaps). Following the $400 \mathrm{MeV}$ upgrade of the Linear Accelerator [15] which injects into the Booster, it was found that intensities up to $5.5 \times 10^{12}$ protons per pulse could be accelerated. Losses in Booster and output beam quality limited useful operation to $5 \times 10^{12}$ and beam quality was improved by operation at lower intensities [16]. Injection of 6 batches at $5 \times 10^{12}$ protons will only provide $3 \times 10^{13}$ protons per Main Injector cycle. ${ }^{2}$ The Fermilab Antiproton Source employed Booster-length Debuncher and Accumulator Rings so increased pbar production depended on higher Main Injector intensity concentrated in one batch length. The capabilities of the neutrino program was limited without enhanced Main Injector beam power.

\section{A. Slip stacking mechanism}

Slip stacking injection allows higher intensity by employing the momentum aperture of the Main Injector to circulate pairs of Booster batches at different momentum, allowing them to slip into alignment for recapture by the accelerating rf waveform. Double batches for pbar production began by using one batch delivered to the central orbit using bucket-to-bucket transfer into a $100 \mathrm{kV}$ rf bucket at the nominal rf frequency in the Main Injector. After decelerating it to a lower momentum (by lowering the rf frequency), a second batch is transferred bucket-to-bucket into an adjacent longitudinal position using a separate $\mathrm{rf}$ system at the injection frequency. The buckets in these $\mathrm{rf}$ systems slip with respect to each other. After the 10th injection, both rf frequencies are increased so that they are symmetric above and below the central orbit frequency. At the moment when the bunches are aligned, the $1 \mathrm{MV}$ acceleration rf system captures both batches in a single

\footnotetext{
${ }^{1}$ The Booster employs harmonic number 84 . For high intensity operation, the Main Injector employs harmonics number 588 using a $53 \mathrm{MHz}$ rf system. A $2.5 \mathrm{MHz}$ system was employed for some pbar operations.

${ }^{2} \mathrm{~A}$ cycle delivering beam to pbar, then NuMI, required gaps for both the rise and fall of the kicker pulse which delivered beam to the pbar target.
}

larger bucket. For multibatch slip stacking, five batches are injected before the deceleration, then five more are added before recapture. The remaining location is filled with a single batch. Figure 4 illustrates this injection process.

Following initial demonstrations of slip stacking, measurements and simulations were carried out [17] which revealed required upgrades and limitations. Beam loading compensation for the Main Injector rf was required for adequate capture in the lower voltage slip stacking buckets [18]. Bunch rotation in the Booster to reduce $\delta p / p$ was also required to match these buckets for bucket-to-bucket transfer. These developments required simulation with ESME [19] and other longitudinal space simulations as well as measurements of Booster and Main Injector beam properties [1]. Measurements of the recaptured beam bunch is shown in Fig. 5. The simulations, along with measured beam properties, documented the requirements for Booster beam properties (see Table I) but also showed that beam loss was expected.

\section{B. Losses from slip stacking}

For sufficiently small emittances, capture efficiencies with slip stacking can be very high. Since the damper system acts on single $53 \mathrm{MHz}$ bunches, it is unsuitable for controlling instabilities during the slipping process. As a result, when bunches are slipping, the instabilities must be controlled by jumping the chromaticity to a large negative value $(-20)$, which results in some beam loss.

Matching the slipping time to the Booster cycle sets the frequency separation required for the counter-slipping bunches. This determines the usable bucket area and sets the longitudinal admittance for slip stacking injection. The momentum acceptance of the Main Injector accepts these two bucket streams, but for the desired intensity, the Booster emittance is a bit too large. This results in various loss issues. Beam which is outside of the slip stacking buckets can move in longitudinal phase such that either (1) it is recaptured in an extraction kicker gap; (2) it has drifted into an injection kicker gap; or (3) at recapture time it is outside of the $1 \mathrm{MV}$ bucket and will not be accelerated.

In addition, when the beam is subjected to high negative chromaticity, the beam lifetime is reduced by transverse loss mechanisms. Control measures for all these losses are now described. ${ }^{3}$

\section{Control of extraction gap loss with antidamping}

Using the wall current monitor, we illustrate the first of these problems with Fig. 6 . In the upper figure we see the 5 NuMI and 1 pbar batches. The pbar production batch is

\footnotetext{
${ }^{3}$ Simulation and measurement showed that the stable bucket area for the first slip stacking rf system was reduced when the second (higher frequency) system was turned on. For operations, the voltage from the second system was not turned on until required for the fifth through tenth injections.
} 

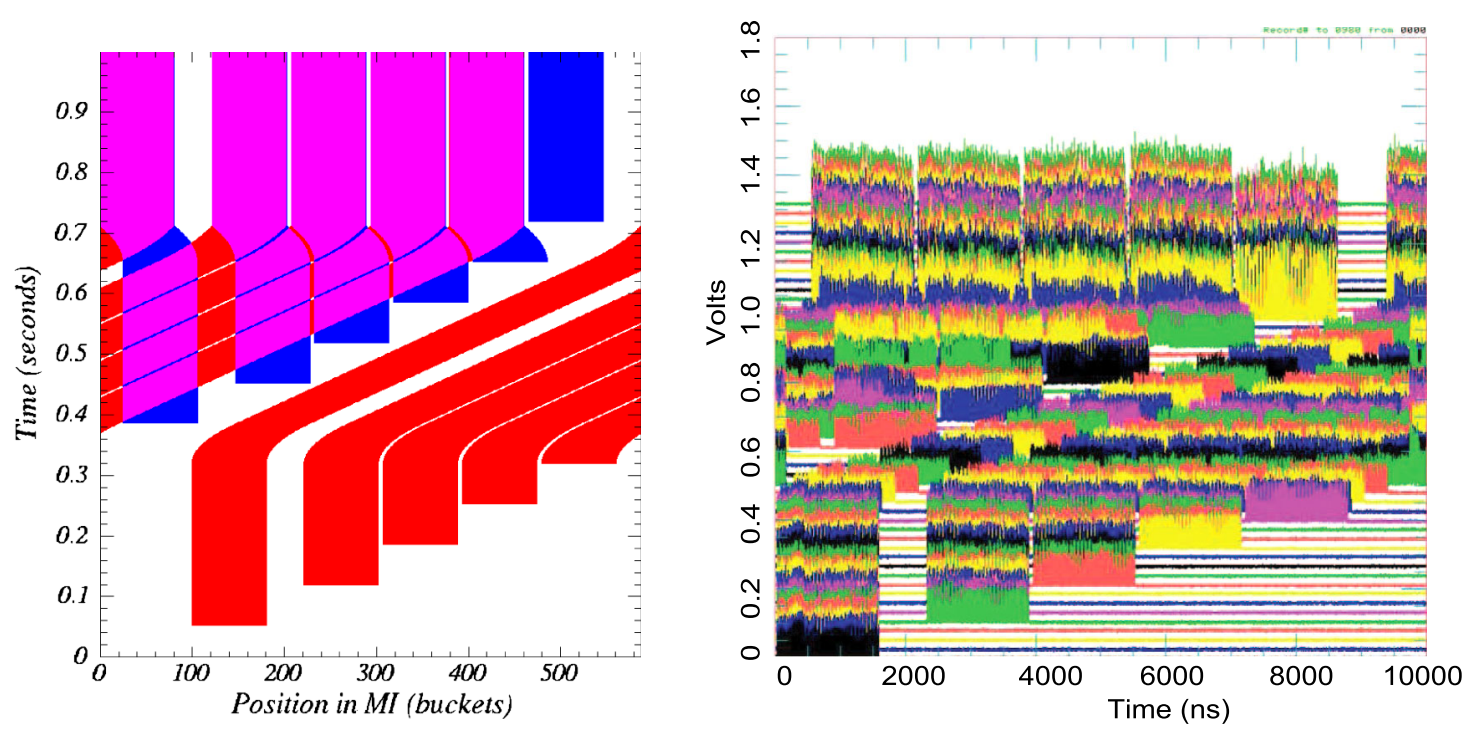

FIG. 4. Left panel shows logic of slip stacking (see Sec. IVA). Five (red) Booster batches are injected into buckets of the first rf system. The frequency is reduced to decelerate them and the next five (blue) batches are injected. When the bunches in the red and blue batches are aligned, the $1 \mathrm{MV}$ rf system captures them. Then the final blue batch is injected. Right panel shows wall current monitor signal during injection for slip stacking operation using 11 Booster batches. Horizontal axis shows time for (nearly) one Main Injector revolution. Main Injector revolution time is 11134 ns at injection. Vertical axis shows bunch intensity with later turns offset vertically. Four double batches followed by one single batch for neutrino production are phased for acceleration at the top of the figure. The first injected batch for pbar production begins at the lower left, slips to the right and is joined by the second pbar injection, arriving at acceleration phase before the top of the figure where it is at the far right. The final neutrino batch arrives following the recapture to the left of the gap for pbar extraction. This panel is from Ref. [1]. With this mode, Main Injector intensities of up to $4.6 \times 10^{13}$ protons per cycle are achieved.

now just to the right of center. We see gaps for the rise (left) and fall (right) of the pbar extraction kicker. These gaps are expanded in the lower panel showing beam that was recaptured and accelerated. Details of the extraction gap beam varies primarily because of variation in the Booster beam momentum distribution. At $8 \mathrm{GeV}$, loss of $1 \%-2 \%$ of the protons is manageable. But after acceleration, losing the same protons at $120 \mathrm{GeV}$ creates more activation near

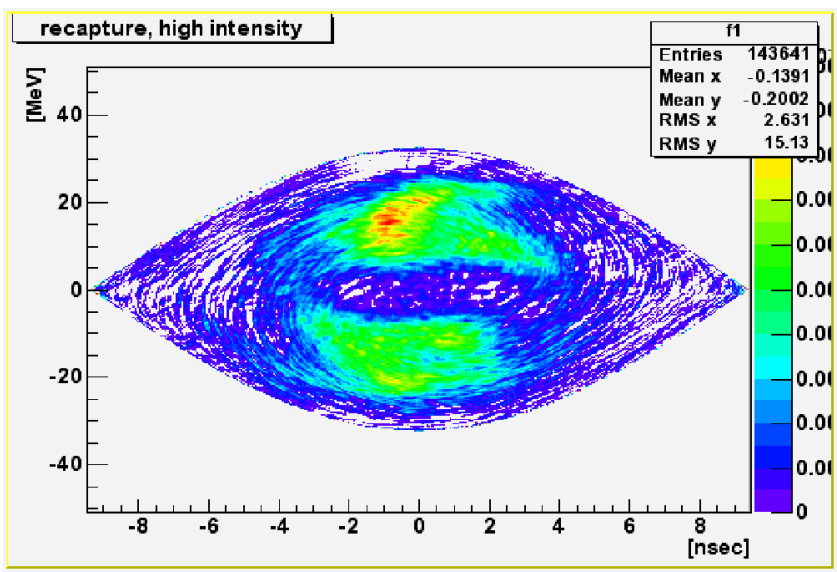

FIG. 5. Tomograph showing momentum offset against time for a recaptured bucket of slip stacked beam as reconstructed from resistive wall monitor signal after creation of $1 \mathrm{MV}$ rf capture bucket. This figure is from Ref. [37].
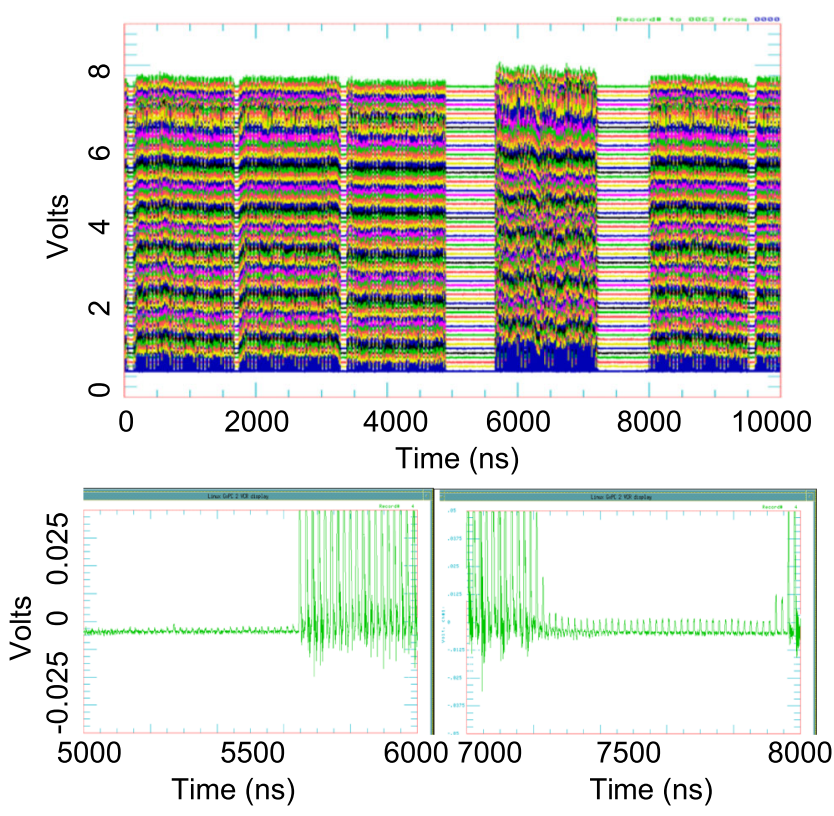

FIG. 6. Wall current monitor plot for slip stacked beam during development of $(5+2)$ slip stacking. For the upper panel, the horizontal axis shows time for (nearly) one Main Injector revolution. Vertical axis shows bunch intensity after recapture with selected turns offset vertically. The lower panel shows portions of one turn, just before extraction, expanded to show the beam captured in the gaps for the rising (left) and falling (right) edges of pbar extraction kicker pulse. This figure is from Ref. [1]. 
extraction devices, which may require maintenance. Removal of these losses is essential.

At low energies, beam bunches in the gaps can be antidamped to achieve removal. Initially this was accomplished by driving the vertical bunch-by-bunch dampers open loop at the fractional machine tune [14]. Later, a vertical kicker was installed near the injection region. Antidamping with this device was rudimentary but effective. A bunch-by-bunch 5 microrad vertical kick at a $\beta_{v}=$ $\sim 50 \mathrm{~m}$ is turned on or off in accordance with an assumed tune near the operating fractional beam tune of $\sim 0.42$. The vertical emittance grows until the protons strike the aperture limit. The assumed tune is programmed in steps of 0.01 applied for 1000 turns. The tunes and steps are modified to optimize beam removal. Much of the proton loss strikes the secondary collimators described below. Loss at the MI522 Lambertson (LAM522) has been significant (see Fig. 14). During slip stacking when there was beam captured in the extraction kicker gaps, losses were capable of exceeding operating loss limits. Loss control by antidamping in these extraction gaps combined with the collimator and gap clearing kicker systems resulted in extended periods with no measurable loss at LAM522.

\section{Control of injection gap loss with gap clearing kickers}

The two rf systems used for slip stacking define separate stable buckets for maintaining bunched beams. Beam outside of those stable buckets will drift longitudinally on the slipping orbits. As discussed in Sec. IV C, beam that is captured in the extraction gaps will create losses. The injection process transfers beam in a series of buckets into the Main Injector using a Lambertson magnet and vertical kicker (K103). Any circulating beam in the ring that passes through the kicker during the injection pulse will be deflected and will strike magnets downstream of the injection kicker in MI104-MI106 (see Figs. 2 and 14). This beam is typically unbunched and is a problem as soon as $1 / 15 \mathrm{sec}$ after it was injected, making antidamping ineffective. The solution was a system of gap clearing kickers (GCK) [20] that are fired to clear the injection gap just prior to the next injection, sending this beam to the Main Injector abort dump. Prior to commissioning the GCK in 2010, the residual radiation buildup in the injection region was minimized by observing limits on the beam loss which was monitored with BLM and residual radiation measurements.

\section{E. Collimation overview}

An additional loss due to slip stacking is from beam that is not captured in the $1 \mathrm{MV}$ rf buckets and thus not accelerated. This beam will follow the momentum offset orbit to lower momentum until the machine aperture is reached. The Main Injector collimation system [21] localizes this loss to limit personnel exposure. It employs a

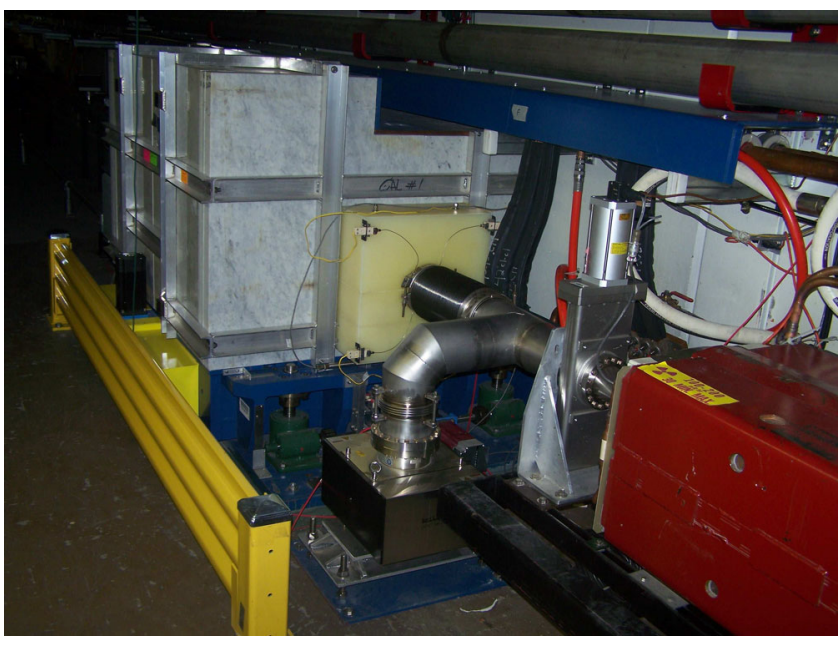

FIG. 7. One of four secondary collimators that employ a thick stainless steel vacuum chamber surrounded by a steel absorber to contain the shower particles. Radiation shielding for personnel is provided by $12 \mathrm{~cm}$ of marble placed on the top, ends, and aisle side of the collimator. At the upstream end a polyethylene block reduces neutron flux to upstream magnet coils. At the downstream end, masks are placed to absorb small angle outscattered particles.

primary-secondary collimator system that defines the momentum aperture with a $0.25 \mathrm{~mm}$ tungsten primary collimator located in a cell (MI230) with normal high dispersion that is just upstream of the dispersion suppressor cells leading to the MI300 straight section. The vertical edge of this collimator is positioned radially inside of the circulating beam to define the momentum aperture. As the beam reaches this aperture it is scattered. Four 20-ton secondary collimators, such as the one shown in Fig. 7, placed at appropriate phase advance downstream, absorb $80 \%$ of the lost beam power with the rest going to nearby devices in the collimation region. Particles that do not experience a sufficient initial scatter may strike the primary collimator 2 or 3 times before being lost from the circulating beam. The loss pattern is distinctive due to the narrow time structure of the unaccelerated $8 \mathrm{GeV}$ beam moving to the low momentum dispersion orbit. Using the time structure as a diagnostic, examination of the ring loss pattern shows that $99 \%$ of the radiation from this beam loss is captured in the collimation region [4]. At Booster intensity of $4.3 \times 10^{12}$ protons per Booster cycle, the incoming momentum spread of the beam results in uncaptured beam loss of about $5 \%$ of the injected beam, resulting in typical lost power of $1.5 \mathrm{~kW}$. This dominant loss is readily measured with the DCCT as shown (for smaller loss) in Fig. 8.

\section{F. Loss control using main injector collimators}

Since the slip stacking process simulation [1] predicted losses due to uncaptured beam, extensive measurements and simulations of the loss process were examined to provide the collimation system design [22]. With appropriate 


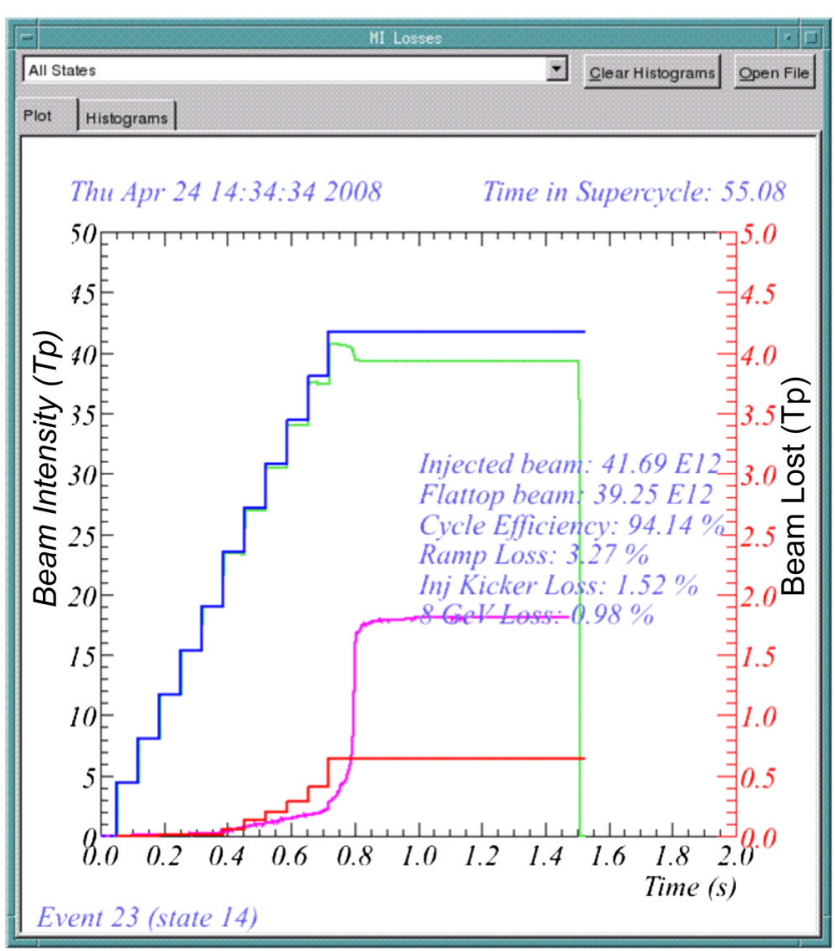

FIG. 8. Typical beam operation for pbar plus NuMI beam production shows the beam intensity vs time in the acceleration cycle. Acceleration begins at $0.755 \mathrm{sec}$. Intensities shown are blue (sum of injected beam), green (circulating beam in Main Injector), red (loss from injection process), and magenta (total loss in ring).

Booster beam emittances and Main Injector rf parameters, the loss patterns were simulated. The time pattern matched measurements but the simulation suggested losses concentrated at locations with high dispersion whereas measurements showed losses concentrated at the Lambertson magnets in the several zero dispersion transfer regions. By including the higher order harmonics of the Main Injector magnets, the simulation could predict these additional loss locations. The simulation indicated that intercepting the uncaptured beam by defining a limiting momentum aperture would allow a collimation system to control the losses. The MARS $[23,24]$ energy deposition code was used to design the secondary collimator systems to provide adequate localization of radioactive isotope production $[25,26]$. Using the output of the tracking simulation as input, radiation issues were evaluated with MARS.

In order to use the secondary collimators, local orbit displacements, timed to impact the beam after $1 \%$ acceleration, permitted uncaptured beam, which had been sufficiently scattered, to strike the collimators. Collimators were positioned while observing aperture requirements for other operating modes. ${ }^{4}$ Measurements following an

\footnotetext{
${ }^{4}$ Large displacements were required since antiproton transfers, using the K304 kicker in the midst of the MI300 straight section, required sufficient aperture for the transfer orbits.
}

extended commissioning phase demonstrated localization of $99 \%$ of the uncaptured beam loss in the collimator region [4]. In addition to absorbing loss from the uncaptured beam, the secondary collimators defined the limiting transverse aperture. As such, the beam removed by antidamping was preferentially lost on them. Additional loss during the slipping process was exhibited by reduced beam life time that was due (in part, at least) to effects of the required large negative chromaticity. These losses also were predominately absorbed in the collimator region. No measurements to separate and quantify the various secondary loss mechanisms were devised but overall loss control showed more than $50 \%$ of loss before acceleration and more than $93 \%$ of loss as acceleration began were well contained in the collimation system.

In Fig. 8 we show beam intensities for a typical Main Injector cycle. One may note the higher injected intensities for the first and sixth injected batches that were directed toward pbar production. With commissioning of the collimation system and availability of control for extraction gap losses, the 11-batch slip stacking process became routine (2008). Intensity was limited by activation of the injection region due to losses in the injection kicker gaps until 2010 when the gap clearing kickers were commissioned. During this time and until the end of the most recent operating period, additional intensity limits came primarily from beam intensity and beam quality from the Booster [27,28]. The losses at the MI8 collimators and emittance monitoring in the MI8 transfer line provided effective monitoring of Booster beam quality. A sequence of Booster improvements, including a major corrector magnet upgrade, allowed steady increases in Main Injector intensity.

\section{APERTURE IMPROVEMENTS}

For intensity increases using slip stacking, studies and simulation found loss mechanisms that must be met with loss control systems (collimation, antidamping). Within the parameters of the Main Injector, these losses could only be localized, not eliminated. It was expected, however, that high intensity would emphasize losses due to aperture limitations that could be eliminated. A series of major and minor efforts were applied to remove, so far as possible, the limits due to these aperture problems.

\section{A. Wide aperture quadrupole}

The transfer regions in the Main Injector occur at straight sections that use regular cells without dipoles. This requires that the three Lambertson magnets for high energy transfers [29] are split with one upstream and two downstream of the intervening quadrupole. The quadrupole center and the Lambertson septum are aligned to the transverse center of the straight section. The circulating and transferring beams must share the quadrupole aperture, thus placing the circulating beam at large displacement. By 


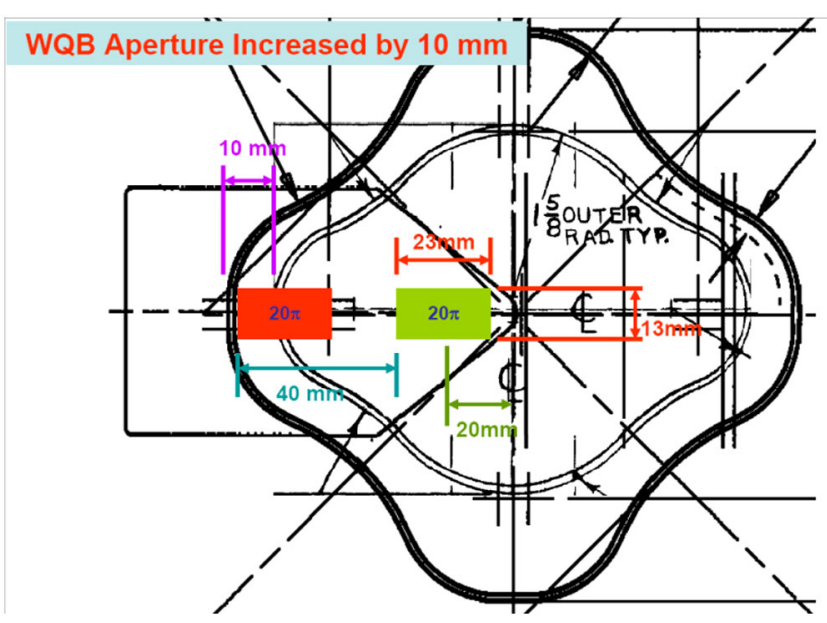

FIG. 9. Aperture improvement using WQB large aperture quadrupoles are shown in this end view of the vacuum pipes (old and new) and the Lambertson that is downstream.

developing a set of wide aperture quadrupoles (WQB [30,31]) with aperture larger by $\sqrt{7 / 4}=1.32$, improved physical aperture and much improved magnetic field quality is available for both beams. Figure 9 shows the new aperture compared with that available before the upgrade. The new beam pipe is illustrated by the "star-shaped" pipe surrounding other features. Beam apertures through the Lambertson magnets are left (circulating) and right (transferred-usually extracted) of center. The beam pipe for the standard quadrupole that was used previously in transfer regions is the smaller star-shaped pipe. The injected beam size is shown with the new range of available positions. WQB magnets were installed at the four high energy transfer locations and three $8 \mathrm{GeV}$ transfer locations in a 2006 facility shutdown.

\section{B. Beam pipe alignment at defocusing quadrupoles}

The elliptical beam pipe used throughout the Main Injector (except at transfer locations as discussed in Sec. VA and others) provides a half aperture of about $23 \mathrm{~mm}$ vertically but more than $58 \mathrm{~mm}$ radially. Assuming full coupling (round beam), the similar maximum $\beta$ values create similar beam aperture requirements: vertically at vertically focusing quadrupoles and radially at horizontally focusing quadrupoles. Even adding a couple of millimeters for momentum aperture requirements, the radial aperture is more generous. During the residual radiation monitoring effort described in Sec. III, a pattern of aperture limitations was observed and understood. The pattern was significant by creating small losses at many locations. The initial observation is documented in Fig. 10. Localized residual radiation was observed on the top of the beam pipe between magnets as shown in Fig. 11. This pattern was found to be due to the flexing of the beam pipe under vacuum load. From a point where it was supported by a bellows (such as that shown in Fig. 12), the pipe

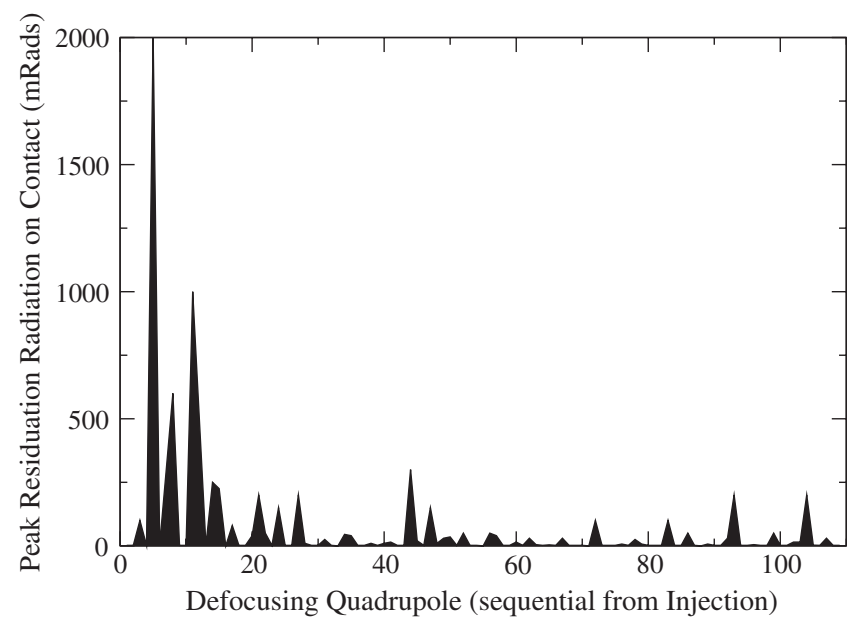

FIG. 10. Residual radiation on contact with top of beam pipe at locations between an upstream dipole and defocusing quadrupole after cool down of a few hours. Data taken on June 11, 2004.

flexed to provide less aperture by about $3 \mathrm{~mm}$ at a point $0.33 \mathrm{~m}$ from the support or $0.5 \mathrm{~m}$ (18 inches) from the upstream dipole. This beam pipe shape created losses where, additionally, the beam pipe for many locations was displaced because it stress-relieved after being inserted through the quadrupole's star-shaped aperture. For reasons not fully understood, this stress relief motion was biased, leaving the beam pipe center line low by $\sim 3 \mathrm{~mm}$ at a fraction of the half cells. The aperture was reduced by $3 \mathrm{~mm}$ from the offset and $1.5 \mathrm{~mm}$ from flexing which created the characteristic localized loss point. Locations with severe offsets were corrected by adding a beam pipe support and realigning the pipe. Collimation in the MI8 line was also helpful.

\section{Other beam pipe alignment issues}

At high beta, the $23 \mathrm{~mm}$ vertical aperture appeared to have several millimeters of clearance from the beam at the three sigma beam boundary. Although a nominal alignment tolerance of $0.25 \mathrm{~mm}$ was applied to magnetic devices, it was expected that beam pipe placement would be adequate with only routine placement at support points. As we explored an unexplained loss downstream of the abort Lambertson magnets, we discovered misalignments up to $6 \mathrm{~mm}$. Proper placement of these beam pipes followed by application of the routine beam steering procedures put the beams on center and greatly reduced the loss in this area. Comparisons of these readings in Figs. 2 and 3 make apparent the improvement.

\section{Bellows installation failure}

The vacuum system is assembled using a series of formed elliptical bellows with rf shielding fingers to pass image currents. A typical bellows is shown in the left panel 

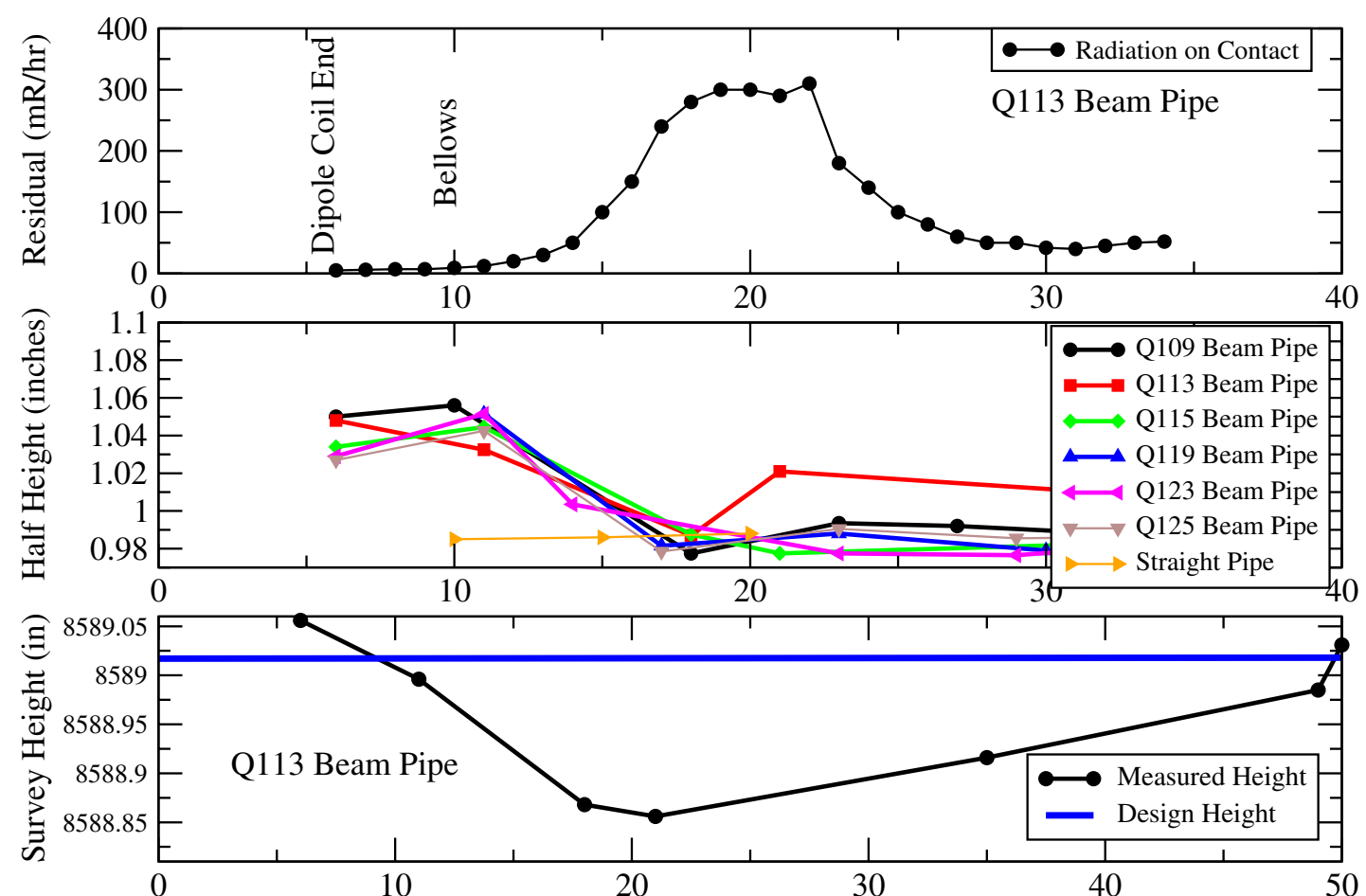

Distance from Dipole Steel Upstream of Vertically Focusing Quadrupoles (inches)

FIG. 11. Geometric distortions of beam pipes permitted beam loss at locations upstream of vertically focusing quadrupoles. The resulting residual radiation distribution on top of beam pipe near Q113 (upper panel); restriction due to beam pipe flexing under vacuum load (center panel) for various locations; and restriction near Q113 from misalignment due to asymmetric stress relieving of insertion-induced beam pipe stress. All locations experienced flexing; many experienced offset.

of Fig. 12. As the loss issues around the ring were addressed by improved tuning, a loss at the 113 loss monitor (LI113) remained. Aperture measurements indicated that the available vertical aperture was reduced compared with other regular cells. Cutting the beam pipe and examining the space from the upstream dipole through Q113 to the downstream dipole revealed a small limitation from beam pipe welding and the bellows problem shown in the right panel of Fig. 12. Replacement of this bellows on March 7, 2011, removed the loss at LI113 as well as the corresponding loss signals at LI114 and LI115. The bellows was misinstalled during a magnet replacement on July 21, 2002, but the loss pattern change was hard to detect until improved instrumentation was available.
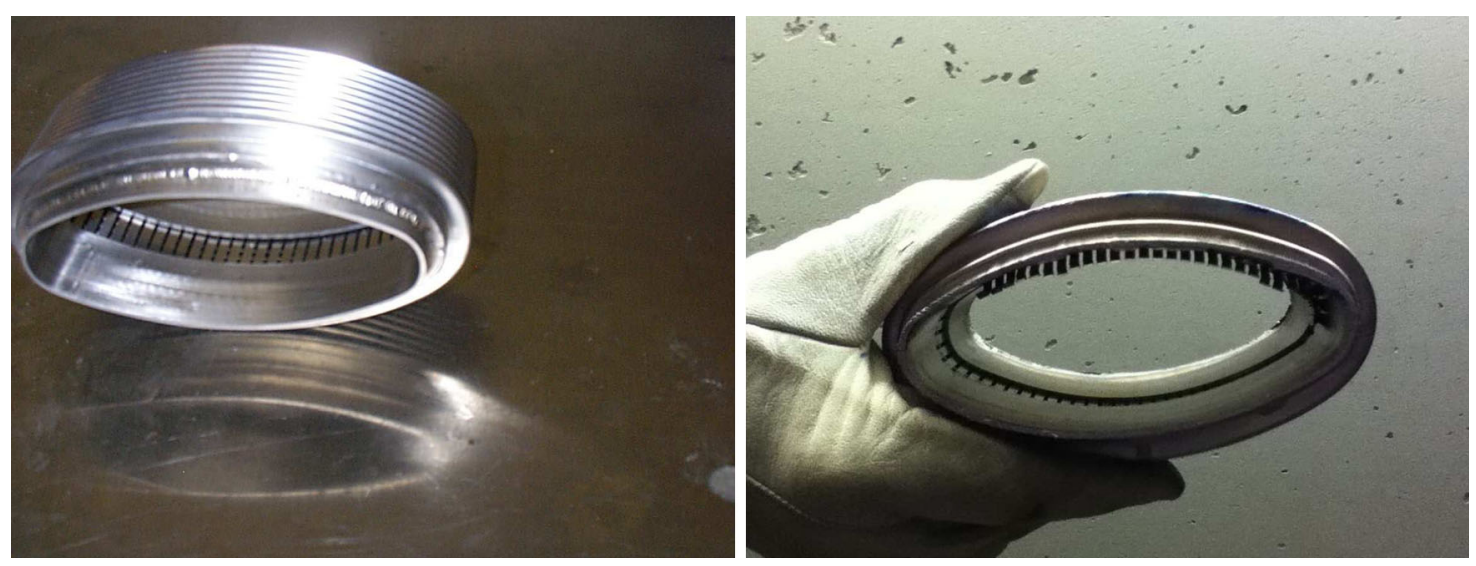

FIG. 12. Elliptical formed bellows used throughout Main Injector to connect beam pipes. Fingers shield beam to provide smooth transition for rf image currents. Failed bellows removed on March 7, 2011. Misinstalled bellows allowed fingers to escape so vertical aperture was reduced by $\sim 5 \mathrm{~mm}$. 


\section{E. Losses from injection tuning errors}

In reviewing the various loss mechanisms, detailed analysis is limited by complexity. In the injection region, in addition to the beam pipe deformations noted above, we also have identified a pattern of losses at phase advances from the injection kicker that indicate injection tuning
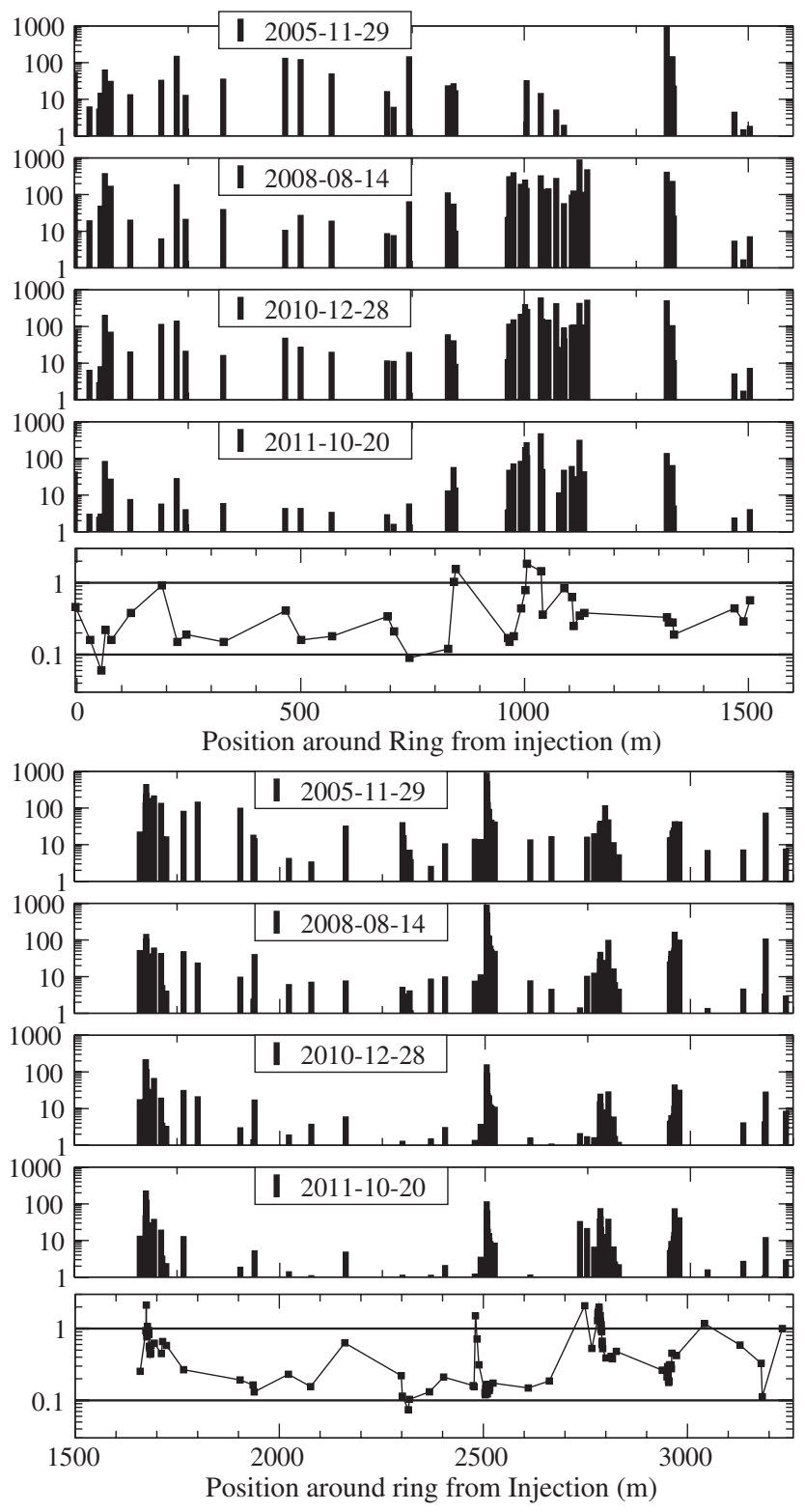

FIG. 13. Main Injector residual radiation history from injection to abort region (upper) and from abort region to injection (lower) measured on contact at barcoded locations. Ratio of measured radiation (November 2011/August 2008) is shown below the measurement sets. Ratio is somewhat overstated due to less cool down time for 2008 data. Major loss points: injection $(50 \mathrm{~m})$, collimation $(1000 \mathrm{~m})$, abort $(1675 \mathrm{~m})$, proton extraction to Tevatron (and other transfers) $(2500 \mathrm{~m})$, NuMI Extraction $(2780 \mathrm{~m})$, antiproton extraction to Tevatron $(2950 \mathrm{~m})$. problems. At phases of $90^{\circ}+n \times 180^{\circ}$ one might see large losses when a kicker misfires, smaller losses when one of the three kicker wave forms are mistimed as well as various loss distributions associated with slip stacked beam in the injection gap (see Sec. IV D). We note that the pipe alignment issues in Sec. VB and the bellows failure in Sec. VD occurred near the vertically focusing Q113. However, this location is also $90^{\circ}+n \times$ $360^{\circ}$ downstream of the injection kicker. Additionally, problems were solved before we acquired the current complement of instrumentation. Losses in the cells downstream of injection as seen in Figs. 2 and 13 include many locations suggestive of injection issues but also a number of locations where some other problem must be responsible. We have been unsuccessful in creating a graphic presentation to illustrate the injection tuning issues but have found that only a small fraction of the losses were from kicker misfires. Although we are documenting many loss issues, a variety of features that were identified in the 2004-2006 radiation surveys were solved without deep understanding.

\section{OTHER LOSS MINIMIZATION EFFORTS}

Tuning to optimally employ the collimation, antidamping, and gap clearing kicker improvements described above continued for a period of several years. The Main Injector specialists and the accelerator operation crew employed the loss display and other tools to progressively limit the locations where significant loss occurred. As a result one could note that when all systems were properly tuned, the major losses occurred early in the cycle and were concentrated in the collimation region and at transfer points. We were now free to examine a limited number of "unexplained" losses.

LAM522 and associated kickers transferred protons to the Tevatron and accepted antiprotons from the Antiproton Source. Despite these complex requirements, control of the slip stacking loss combined with careful tuning resulted in loss-free operation for extended time periods. A campaign to tune more carefully at other transfer locations reduced losses at each of them but some loss remained.

The improved sensitivity provided by new BPM's facilitated some studies that required the better resolution. In doing these measurements, we discovered a BPM detector that had an intermittent faulty connection. This error had resulted in setting the orbit to wrong position by up to $15 \mathrm{~mm}$. The large horizontal aperture of the Main Injector allowed adequate transmission despite such errors but losses improved when this was corrected. Occasional other BPM failures were quickly noted after implementation of the beam loss display. Occasional BLM failures also allowed some additional activation before they were identified. 


\section{OPERATIONAL LOSS CONTROL: ABORTS AND INHIBITS}

The beam power of the Main Injector, especially at high energy, is sufficient to create damage in a single pulse. Additionally, environmental concerns in the transport line to the NuMI target demand very high beam quality and beam transport control [32]. The beam abort system at MI40 can be employed to kick all beam out in one turn. The abort system tracks the proton energy and sets the kickers and transport line magnets to properly deliver the beam to the abort beam absorber. Each beam loss monitor channel can trigger the beam abort. The integral loss for the acceleration cycle and the loss in a $39 \mathrm{~ms}$ running sum are compared to abort thresholds. The abort threshold is set separately for each BLM channel, sum type, and machine operating mode. Special abort triggers have been created for the NuMI operation that monitors the status of the rf accelerating system and beam positions to protect the NuMI beam line [33]. An abort inhibits beam for subsequent cycles until it is reset by an operator. Only an occasional device failure has caused these systems to be required to protect the facilities.

To avoid beam loss from system or device failures, the beam injection is inhibited based on examination of the status of the beam permit. This inhibit is applied at the upstream end of the linear accelerator. A variety of inputs to this system inhibit further operation until reset including status inputs from vacuum, power supply, rf, and other accelerator systems. As further protection, audible alarms that require operator reset are triggered for various offnormal states. During regular operation the most common alarm is due to the beam energy loss signal constructed by the BEAMS front end [10] that sums the beam energy loss calculated by multiplying the incremental beam loss by the beam energy. The threshold for this alarm was adjusted (as improvements permitted) to match the capability of the Main Injector and Booster when all systems were operating well at high intensity. These alarms were addressed by adjusting parameters in one of the machines, by identifying and repairing system failures, or by reducing intensity until high quality operation could be restored. By observing limits on losses, we achieved higher intensities while reducing machine component activation.

\section{RESULTS}

We demonstrate the success in controlling and localizing loss by examining the loss display and by reviewing the history of residual radiation measurements.

\section{A. Beam loss monitor display}

As a measure of the successful loss control efforts, compare Fig. 3 with Fig. 2. The injection gap losses (top row8th through 10th loss monitors-green brace) have been addressed by the gap clearing kickers. The losses at the 17th through 19th monitors were eliminated by replacing the faulty bellows near Q113. The loss in the collimation region (second row-first 20 loss monitors-cyan brace) is distributed in a more favorable way, emphasizing the 1st and 2nd secondary collimators. Loss at the abort area (second row-48th through 58th monitors-yellow brace) is reduced. Loss at the Recycler transfer points is eliminated (red brace near end of first row) or greatly reduced (red brace at center of second row). Loss at LAM522 region is eliminated (purple brace at end of third row - 48th through 56th monitors) since the remaining bar is due to a BLM pedestal offset. In the fourth row we see loss at LAM608 (NuMI extraction-purple brace at 12th through 22nd monitor) and LAM620 (pbar transfer - purple brace at 38th through 43rd monitors) are significantly reduced. Fewer signals are seen throughout the ring while a few of the remaining bars are due to pedestal offset in the BLM electronics. With this fairly clean display, changes in the loss profile provided an alert to the operations crew.

For loss reduction, the calibration of the BLM system in Rads at the BLM detector is sufficient since we wish to eliminate any observable loss. For BLM calibration in protons lost, we are aware that each loss monitor has a relation to the number of protons lost that is in principle dependent upon the exact loss mechanism details, including the beam orbit and to the local geometry of the machine components. In most of the ring, we position BLM sensors on the outer wall of the enclosure above the beam line height at the downstream end of each quadrupole that provides a degree of uniformity for the response. Constraining the orbit by requiring good transmission leaves little room for a change of sensitivity. Changes in loss are due primarily to beam quality. We also note that losses are nearly local but, almost everywhere, a significant loss in one detector will also create a response in a nearby detector. Detailed measurements to relate lost protons to BLM signals had mixed results and are not employed in results for this document. Geometric oversampling due to placing many more BLM's at the transfer points could cause a distortion but operationally the losses are sufficiently concentrated that the oversampling has little impact on overall loss evaluation. We provided guidance on the impact of the collimation system in Sec. IV F but that assumed that all loss monitors had the same response to a lost proton. We believe that is a conservative estimate since we are certain that the collimators shield the collimation region loss monitors to make them provide smaller response to proton loss. Available data suggest that loss monitors have similar calibrations in lost protons within a factor of 2 .

\section{B. Summary of residual radiation monitoring}

The definitive measure of loss control is reduced residual radioactivity for hands-on maintenance and upgrade activities. Losses cannot be distributed uniformly. 
This localization of the loss implies that no single measure of radiation reduction will describe the impact of improvements on the $3.3 \mathrm{~km}$ scale of the Main Injector. The successes of the loss control campaign in the Main Injector has lead to enormous improvements in all regions except at the collimators. Figure 13 provides snapshots of the residual radiation at barcoded locations selected from more than 50 such data sets. Note the logarithmic scale where a reduction by a factor of 10 shows with a reduction of a bar by $1 / 3$ of the vertical scale. We see, as we did with the loss display, the residual radiation is greatly reduced. Only the collimator region remains at a nearly constant residual rate. Detailed comparisons are best done as discussed in Sec. VIIIC since the data were recorded with various delays between beam activation and measurement.

\section{Residual radiation history}

An alternate display of the data in Sec. VIII B is available by examining the residual radiation history provided by the measurements at barcoded locations around the ring [12]. Selected locations are illustrated in Fig. 14. The expected correlation between loss and residual radiation has been established [34]. Linear fits to the correlation between half life weighted beam loss and residual radiation history are applied using three or four isotopes. A description that is adequate for most planning purposes is achieved with three isotopes of manganese: ${ }^{54} \mathrm{Mn},{ }^{52} \mathrm{Mn}$, and ${ }^{56} \mathrm{Mn}$ having half life values of 312.3 days, 5.591 days, and 2.58 hours. ${ }^{59} \mathrm{Fe}$ (44.5 days) or ${ }^{51} \mathrm{Cr}$ (27.7 days) improves some fits.

We show measurements and fit results for the upstream end of the Lambertson Septum LAM522 where losses during early $(5+2)$ slip stack operation resulted in very high radiation levels. Improved tuning followed by implementation of the antidamping for the extraction gaps reduced the loss so the residual levels began to fall. Later we achieved loss-free operation at this location.

The losses from the injection gap impacted devices in several half cell locations downstream from the injection kicker (K103). We illustrate this with the history at Q104 Downstream. Losses were monitored and beam intensity was limited to keep the residual radiation at a level suitable to permit tunnel modifications during the 2009 facility shutdown. At this time, the GCK magnets were installed but the power supplies and cables awaited additional tunnel time. The peak in radiation following the 2009 shutdown was the result of relaxed requirements on beam loss monitor values. GCK commissioning followed the 2010 shutdown and after successful commissioning, the loss in this region dropped to small values.

As another example, we show the loss history at the S408 sextupole. Detailed measurements at this location (and several others) revealed radial loss. Examination of the BLM signals frequently showed loss at transition although other loss times contributed. This was a situation
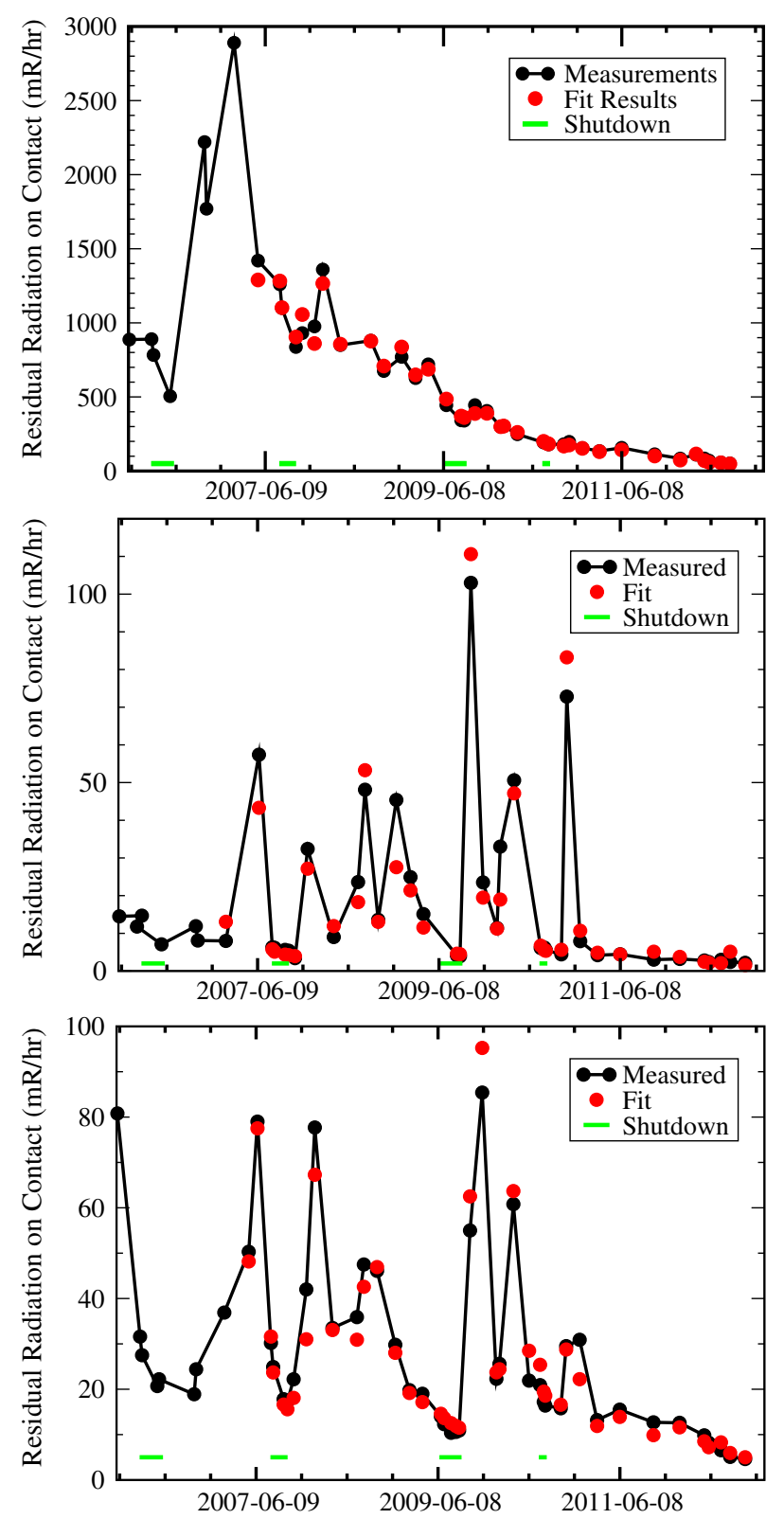

FIG. 14. Residual Radiation History at barcoded locations fitted to BLM loss weighted by set of isotope half lives. LAM522 fit to LI522A loss (top), Top of Q104 Downstream End fitted to LI104 loss (center) and Top of Sextupole S408 fitted to LI408 loss (bottom). Reduced loss at Q104DS followed commissioning of Gap Clearing Kickers.

where the beam loss monitor display proved very helpful. Significant radiation issues can build up with only a small impact on transmission. As other problems were addressed, this loss point received appropriate attention and losses were mitigated.

\section{SUMMARY OF HIGH INTENSITY OPERATION}

The beam properties achieved for high intensity operation of the Main Injector matched the goals set in the 
Proton Plan [3]. They are summarized in Table I. When operating to maximize both neutrino and pbar production, we employed the $9+2$ slip stacking mode described above (mixed mode) at the $2.2 \mathrm{~s}$ repetition rate achievable. The high energy physics program required additional operating modes. The Main Injector (as its name suggests) is the source of $150 \mathrm{GeV}$ protons and antiprotons for the Tevatron Collider. The transfer of $150 \mathrm{GeV}$ beam and setup for that required interruptions to the mixed mode operation. More frequently the process was interrupted to transfer antiprotons from the Accumulator to the Recycler. Both of these processes were gradually optimized to improve high intensity productivity. A portion of the Main Injector time was devoted to slow spill operation which provided $4.05 \mathrm{sec}$ of extraction at $120 \mathrm{GeV}$ for the test beam and fixed-target experimental program. When the NuMI beam line was unavailable to take beam, a pbar-only mode with two slip stacked batches was operated. Since the collection and cooling power of the Antiproton Source was saturated by the standard $2.2 \mathrm{sec}$ repetition rate, the pbar-only mode repetition rate was not maximized. During the brief intervals when the Tevatron or Antiproton Source were unavailable, a NuMI-only mode with 11 batch slip stacking was employed. At the end of the Tevatron run, a 9 batch slip stacking mode with $2.066 \mathrm{sec}$ cycle time was created to maximize beam power while observing a per pulse intensity limit of $3.75 \times 10^{13}$ protons per pulse designed to protect the neutrino production target from thermal shocks.

In Fig. 15 we document the weekly sum of protons accelerated to $120 \mathrm{GeV}$ from 2004 through 2012. With a typical intensity of $4.2 \times 10^{13}$ protons per cycle, the maximum weekly sum is $1.15 \times 10^{19}$ protons/week. We find that by the beginning of 2008 , we were achieving up to $50 \%$ of this rate but by commissioning the 11 batch slip stacking and the collimation, we achieved $70 \%$ of this

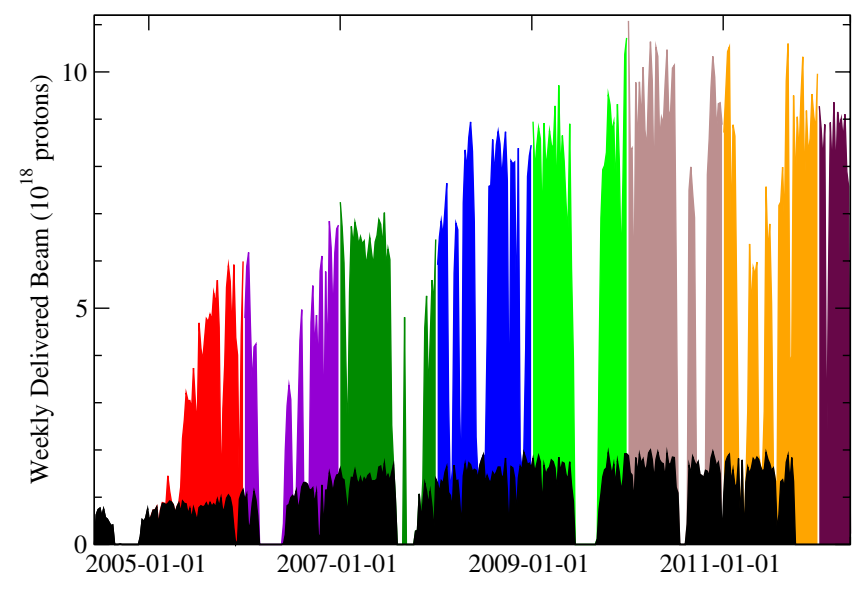

FIG. 15. Weekly Summary of Protons Accelerated in Main Injector for delivery to the pbar and NuMI targets. This stacked bar chart shows pbar weekly beam in black bars with colored bars indicating the NuMI weekly beam (each year a new color) so the top of the bar indicates the total weekly delivered $120 \mathrm{GeV}$ beam. mark during 16 weeks of that year. Steady progress in 2009 was culminated with two weeks that achieved $90 \%$ of that target. In 2010 there were 8 weeks above $88 \%$ of that target. That year we achieved our peak weekly integrated beam of $1.109 \times 10^{19}$ protons per week or $96.4 \%$ of this goal.

These beam power limitations for the Main Injector are principally set by the capabilities of the Fermilab Booster in combination with design properties of the Main Injector. The momentum aperture of the Main Injector is adequate for slip stacking injection. The Booster 15 hertz structure sets a requirement for slipping speed and thereby for frequency difference for the two slip stacking rf systems. The injection buckets created by $100 \mathrm{kV}$ rf systems have as large an acceptance as possible without overlapping the buckets. The longitudinal emittance and especially the (related) momentum spread of the Booster beam does not match these buckets at the desired intensity. The uncaptured beam loss in high intensity operation is due to Booster beam with too large $\delta p / p$. Additional losses in the injection and extraction gaps are also dictated by Booster emittance in combination with bunch-to-bunch phase offsets due to coupled bunch instabilities in the Booster. Additional losses due to large negative chromaticity operation are observed but are small. These also would likely be smaller with lower Booster transverse emittance and would not be a problem if slip stacking injection were not required. In summary, using the current equipment, the intensity capability of the Main Injector is not challenged until a more intense injector is available..$^{5}$

\section{CONCLUSIONS AND OBSERVATIONS}

The requirement to produce abundant antiprotons and neutrinos to match Fermilab's High Energy Physics program required enhancements to the initial Main Injector configuration. The Proton Plan [3] as developed in 20042006 identified and addressed the limitations in the Main Injector. Improvements to the Fermilab Booster were also addressed. The results included improved beam properties as well as lower losses in the Booster. The performance envelope of the Booster continues to define the intensity limit for Main Injector operation.

Main Injector loss control efforts have been directed at maintaining low residual radiation for maintenance and upgrade activities. This has focused efforts on optimizing the use of the Main Injector collimation system and in vigilant attention to removing localized loss points since even a fairly small loss will allow accumulation of residual radiation that can impact planning for repairs.

The 2012-2013 facility shutdown will implement a series of modifications designed to permit operation of the Main Injector at $700 \mathrm{~kW}$ beam power at $120 \mathrm{GeV}$

\footnotetext{
${ }^{5} \mathrm{~A}$ limit due to $\mathrm{rf}$ capability is expected at intensities about $30 \%$ higher than the current per pulse operation [35].
} 
[36]. This will be achieved with modest improvements in per cycle beam intensity and by enhancing the repetition rate from $2.2 \mathrm{sec}$ to $1.3 \mathrm{sec}$ by employing the Recycler Ring as an $8 \mathrm{GeV}$ stacking ring. Twelve batch slip stacking in the Recycler will be followed by recapture in the 1-MV Main Injector rf system. When Recycler modifications and Booster repetition rate enhancements $[27,28]$ are complete we expect operation at $700 \mathrm{~kW}$ to be available. We are expecting radiation issues to increase only proportional to the beam power.

\section{ACKNOWLEDGMENTS}

The high intensity operation of the Main Injector was accomplished by the efforts of the entire Fermilab Accelerator Division. We thank the many helpful people in the other machine departments and support departments and the Operations Department for their ongoing commissioning and tuning efforts. The Fermilab Technical Division created many devices and provided support for maintenance and upgrade activities. We thank the Fermilab experimenters for their support and encouragement. The Fermi National Accelerator Laboratory is operated by Fermi Research Alliance, LLC under Contract No. DE-AC0207CH11359 with the United States Department of Energy.

[1] K. Seiya, T. Berenc, B. Chase, J. Dey, P. Joireman, I. Kourbanis, and J. Reid, in Proceedings of the 2007 Particle Accelerator Conference, edited by C. Petit-JeanGenaz (IEEE, Piscataway, NJ, 2007), pp. 742-744, DOI:10.1109/PAC.2007.4440760.

[2] Eric Prebys et al., Fermilab Report No. Beams-doc 1441, 2004 [http://beamdocs.fnal.gov/AD-public/DocDB/ ShowDocument?docid=1441].

[3] Proton Plan Design Handbook (Fermilab, Batavia, IL, 2006) [http://www-accel-proj.fnal.gov/Proton_Plan/Design_ Handbook/index.shtml].

[4] Bruce Brown, Philip Adamson, David Capista, A. I. Drozhdin, David E. Johnson, Ioanis Kourbanis, Nikolai V. Mokhov, Denton K. Morris, Igor Rakhno, Kiyomi Seiya, Vladimir Sidorov, Guan Hong Wu, and Ming-Jen Yang, in Proceedings of the 23rd Particle Accelerator Conference, 2009, Vancouver, British Columbia, Canada, edited by Martin Comyn (2009), pp. 28412843 [http://accelconf.web.cern.ch/AccelConf/PAC2009/ papers/we6rfp025.pdf].

[5] The Fermilab Main Injector Technical Design Handbook (Fermilab, Batavia, IL, 1994) [http://inspirehep .net/record/1219664/files/fermilab-design-1994-01.pdf], Fermilab Report No. FERMILAB-DESIGN-1994-01.

[6] The loss control section is expanded from Bruce $\mathrm{C}$. Brown, in Proceedings of the 52nd ICFA Advanced Beam Dynamics Workshop on High-Intensity and HighBrightness Hadron Beams (HB2012), Beijing, 2012 (2012), pp. 264-268 [http://accelconf.web.cern.ch/ AccelConf/HB2012/papers/tuo1b04.pdf].

[7] B. Banerjee et al., AIP Conf. Proc. 868, 540 (2006).
[8] R.E. Shafer, R.E. Gerig, A.E. Baumbaugh, and C.R. Wegner, in Proceedings of the 12th International Conference On High-Energy Accelerators, edited by Francis T. Cole and Rene Donaldson (Fermilab, Batavia, IL, 1983), pp. 609-615 [http://inspirehep.net/record/ 263539? ln=en].

[9] A. Baumbaugh et al., JINST 6, T11006 (2011).

[10] The beam current monitor software described in T. Meyer, D. Slimmer, and D. Voy, JINST 6, T11004 (2011).

[11] Bruce C. Brown and Guan H. Wu, Fermilab Report No. Beams-doc 3299 v2, 2009 [http://beamdocs.fnal.gov/ AD-public/DocDB/ShowDocument?docid=3299].

[12] Bruce C. Brown, Fermilab Report No. Beams-doc 3523 v1, 2009 [http://beamdocs.fnal.gov/AD-public/DocDB/ ShowDocument?docid=3523].

[13] B. C. Brown, D. P. Capista, Ioanis Kourbanis, Nikolai V. Mokhov, and Vladimir Sidorov, in Proceedings of the 2007 Particle Accelerator Conference, edited by $\mathrm{C}$. Petit-Jean-Genaz (IEEE, Piscataway, NJ, 2007), p. 1673, DOI:10.1109/PAC.2007.4440860.

[14] P. Adamson, W. J. Ashmanskas, G. W. Foster, S. Hansen, A. Marchionni, D. Nicklaus, A. Semenov, D. Wildman, and H. Kang, in Proceedings of the 2005 Particle Accelerator Conference, edited by C. Horak (IEEE, Piscataway, NJ, 2005), pp. 1440-1442, DOI:10.1109/ PAC.2005.1590791.

[15] K. Junck, L. Allen, Thomas K. Kroc, J. MacLachlan, Elliott S. McCrory, A. Moretti, R. Noble, M. Popovic, and C. Schmidt, in Proceedings of the 4th European Particle Accelerator Conference London, 1994, edited by Vic P. Suller and Christine Petit-Jean-Genaz (World Scientific, New York, 1994), pp. 751-753 [http://accelconf.web.cern.ch/AccelConf/e94/PDF/ EPAC1994_0751.PDF].

[16] R. C. Webber, in Proceedings of 7th ICFA Miniworkshop on High Intensity High Brightness Hadron Beams: Beam Halo and Scraping, edited by Nikolai V. Mokhov and Weiren Chou (Fermilab, Batavia, Ill., 1999), pp. 89-95.

[17] K. Seiya et al., in Proceedings of 2005 Particle Accelerator Conference, Knoxville, Tennessee, edited by C. Horak (IEEE, Piscataway, NJ, 2005), pp. 347-349, DOI:10.1109/PAC.2005.1590430.

[18] J. Dey and I. Kourbanis, in Proceedings of 2005 Particle Accelerator Conference, Knoxville, Tennessee, edited by C. Horak (IEEE, Piscataway, NJ, 2005), pp. 1958-1960, DOI:10.1109/PAC.2005.1590972.

[19] J.A. MacLachlan, Users Guide to ESME, Technical Report (Fermilab, Batavia, IL, 2000); Report No. FERMILAB-TM-2132, November 2000. ESME software, http://www-ap.fnal.gov/ESME/.

[20] I. Kourbanis, P. Adamson, J. Biggs, B.C. Brown, D. Capista, C. C. Jensen, G. E. Krafczyk, D. K Morris, D. Scott, K. Seiya, S. R. Ward, G. Wu, and M.-J. Yang, in Proceedings of the 2011 Particle Accelerator Conference, edited by Todd Satogata and Kevin Brown (New York, 2011), pp. 1870-1872 [http://accelconf.web.cern.ch/ AccelConf/PAC2011/papers/wep205.pdf].

[21] Bruce C. Brown, in Proceedings of the 42nd ICFA Advanced Beam Dynamics Workshop on High-Intensity, 
High-Brightness Hadron Beams (HB2008), Nashville, $T N$, edited by Stuart Henderson (JACoW, 2008), p. 312 [http://accelconf.web.cern.ch/AccelConf/HB2008/papers/ wgc11.pdf].

[22] Alexandr I. Drozhdin, Bruce Brown, David E. Johnson, Ioanis Kourbanis, Nikolai V. Mokhov, I. Rakhno, Vladimir Sidorov, and Kiyomi Koba, in Proceedings of the 2007 Particle Accelerator Conference, edited by C. Petit-Jean-Genaz (IEEE, Piscataway, NJ, 2007), p. 1688, DOI:10.1109/PAC.2007.4440865.

[23] Nikolai V. Mokhov, The MARS Code System User's Guide Version 13(95) (Fermilab, Batavia, IL, 1995), FN 628.

[24] N. V. Mokhov and S. I. Striganov, AIP Conf. Proc. 896, 50 (2007).

[25] Igor Rakhno, Fermilab Report No. TM 2391-AD, 2007.

[26] Igor Rakhno, in Proceedings of the 9th Workshop on Shielding Aspects of Accelerators, Targets and Irradiation Facilities (SATIF-9) 2008, Oak Ridge, Tennessee (2008).

[27] F. G. Garcia and W. Pellico (PIP Collaboration), in Proceedings of the 52nd ICFA Advanced Beam Dynamics Workshop on High-Intensity and High-Brightness Hadron Beams (HB2012), Beijing, 2012 (2012), pp. 320-323 [http://accelconf.web.cern.ch/AccelConf/HB2012/papers/ tuo3c02.pdf]

[28] F. G. Garcia and W. Pellico (PIP Collaboration), in Proceedings of the 52nd ICFA Advanced Beam Dynamics Workshop on High-Intensity and HighBrightness Hadron Beams (HB2012), Beijing (2012), pp. 378-380 [http://accelconf.web.cern.ch/AccelConf/ HB2012/papers/weo3a04.pdf].

[29] D. E. Johnson, R. Baiod, D. J. Harding, P. S. Martin, and M. May, in Proceedings of the 1995 IEEE Particle Accelerator Conference, Dallas, 1995 (IEEE, New York, 1995), p. 1346, DOI:10.1109/PAC.1995.505217.

[30] David J. Harding, Leon Bartelson et al., in Proceedings of the 2007 Particle Accelerator Conference, edited by
C. Petit-Jean-Genaz (IEEE, Piscataway, NJ, 2007), p. 455, DOI:10.1109/PAC.2007.4440243.

[31] Weiren Chou, Leon Bartelson et al., in Proceedings of the 2007 Particle Accelerator Conference, edited by C. PetitJean-Genaz (IEEE, Piscataway, NJ, 2007), p. 1685, DOI:10.1109/PAC.2007.4440864.

[32] S.C. Childress, in Proceedings of the 42nd ICFA Advanced Beam Dynamics Workshop on High-Intensity, High-Brightness Hadron Beams (HB2008), Nashville, TN, edited by Stuart Henderson (JACoW, 2008), pp. 475-478 [http://accelconf.web.cern.ch/AccelConf/HB2008/papers/ wgf12.pdf].

[33] S.C. Childress, in Proceedings of the 42nd ICFA Advanced Beam Dynamics Workshop on High-Intensity, High-Brightness Hadron Beams (HB2008), Nashville, TN, edited by Stuart Henderson (JACoW, 2008), pp. 379-381 [http://accelconf.web.cern.ch/AccelConf/HB2008/papers/ wgd12.pdf].

[34] Bruce C. Brown and Guan Hong Wu, in Proceedings of the 46th ICFA Advanced Beam Dynamics Workshop on High-Intensity and High-Brightness Hadron Beams (HB2010), edited by Jan Chrin (Morschach, Switzerland, 2010), pp. 391-394 [http://accelconf.web.cern.ch/ AccelConf/HB2010/papers/tuo2c04.pdf].

[35] Ioanis Kourbanis, Fermilab Report No. Beams-doc 1927 v1, 2005 [http://beamdocs.fnal.gov/AD-public/DocDB/ ShowDocument?docid=1927].

[36] Paul Derwent, in Proceedings of the 3rd International Particle Accelerator Conference, New Orleans, Louisiana, USA (2012), pp. 4185-4189 [http://accelconf .web.cern.ch/AccelConf/IPAC2012/papers/frybp01.pdf].

[37] K. Seiya, T. Berenc, B. Chase, W. Chou, J. Dey et al., in Proceedings of the 39th ICFA Advanced Beam Dynamics Workshop on High Intensity High Brightness Hadron Beams 2006 (HB2006), Tsukuba, Japan (2006), pp. 293-297 [http://accelconf.web.cern.ch/AccelConf/ abdwhb06/PAPERS/THAY02.PDF]. 